\title{
Dynamics across the structural transitions at elevated temperatures in $\mathrm{Na}_{0.7} \mathrm{CoO}_{2}$
}

Fanni Juranyi ${ }^{1}$, a , Martin Månsson ${ }^{1,2}$, Jorge L. Gavilano ${ }^{1}$, Mattia Mena ${ }^{1}$, Ekaterina Pomjakushina ${ }^{3}$, Marisa Medarde ${ }^{3}$, Jun Sugiyama $^{4}$, Kazuya Kamazawa ${ }^{5}$, Bertram Batlogg ${ }^{6}$, Hans R. Ott ${ }^{6}$ and Tilo Seydel ${ }^{7}$

${ }^{1}$ Laboratory for Neutron Scattering and Imaging, Paul Scherrer Institute, Villigen PSI, Switzerland

${ }^{2}$ Laboratory for Quantum Magnetism, EPF Lausanne, Switzerland

${ }^{3}$ Laboratory for Development and Methods, Paul Scherrer Institute, Villigen PSI, Switzerland

${ }^{4}$ Toyota Central Research and Development Laboratories, Inc., Nagakute, Aichi, Japan

${ }^{5}$ Comprehensive Research Organisation for Science and Society, Tokai, Ibaraki, Japan

${ }^{6}$ Laboratory for Solid State Physics, ETH Zürich, Zürich, Switzerland

${ }^{7}$ Institut Laue-Langevin, Grenoble, France

\begin{abstract}
The layered transition-metal oxide $\mathrm{Na}_{\mathrm{x}} \mathrm{CoO}_{2}$ has been studied extensively both for its correlated electronic properties as well as for potential battery applications. It was discovered that high-temperature $\mathrm{Na}$ ion vacancy order and dynamics can be very useful to tailor low-temperature properties of members of this compound family. We have studied the Na-ion dynamics on the atomic length-scale in the $\mathrm{Na}_{0.7} \mathrm{CoO}_{2}$ compound by neutron spectroscopy. The temperature dependence of both the elastic and the inelastic intensities show steps at $T_{\mathrm{A}} \approx 290 \mathrm{~K}$ and $T_{\mathrm{B}} \approx 400 \mathrm{~K}$. At $T_{\mathrm{A}}$ the step is shown to be connected to low energy phonons, while at $T_{\mathrm{B}}$ the $\mathrm{Na}$ ion diffusion suddenly gets fast enough, and the characteristic signal of quasielastic scattering appears. The current results further elucidate the subtle changes in the $\mathrm{Na}$ ion dynamics that have been revealed in our previous neutron diffraction studies [1], intimately connecting structural transformations at $T_{\mathrm{A}}$ and $T_{\mathrm{B}}$ with the opening-up of $1 \mathrm{D}$ and 2D Na-ion diffusion paths. Finally, the estimated diffusion coefficient above $T_{\mathrm{B}}$ was found to differ from the one measured by muon-spin relaxation $\left(\mu^{+} \mathrm{SR}\right)$ [2] by about four orders of magnitude. However it might be that the present QENS data rather describe a fast localized prozess than a long range translational diffusion. Within this model the corresponding time scale $(\hbar / E)$ would be in the order of $50 \mathrm{ps}$.
\end{abstract}

\section{Introduction}

The fast development of rechargeable lithium-ion batteries is clearly one of the biggest success stories for modern electrochemistry. However, recently the future for Li-ion technology has been questioned due to the limited resources of lithium metal along with its large negative environmental impact and high cost during extraction from the earth's crust. As a result, possible replacement candidates based on sodium, that is much more abundant than lithium, are becoming more and more interesting for battery researchers [3]. In that context, the $\mathrm{Na}_{\mathrm{x}} \mathrm{CoO}_{2}$ compound is in many ways the Na-analog of the most common Li-ion battery electrode $\mathrm{Li}_{\mathrm{x}} \mathrm{CoO}_{2}$. But, in addition, $\mathrm{Na}_{\mathrm{x}} \mathrm{CoO}_{2}$ is also interesting on its own right. It has a rich phase-diagram displaying superconducting, magnetic, as well as thermoelectric phases that strongly depend on the Na-content $(x)$. Furthermore, the potential landscape from ordered $\mathrm{Na}-$ vacancies $[4,5]$ as well as room-temperature Na-dynamics $[6,7]$ have been shown to play a very important role in the formation of the ground state of this material. This has opened up intriguing possibilities for tuning fundamental physical properties [8] by controlling the dynamic processes and through that the self-assembling structure on a sub-nanoscale. Consequently, for both, applied as well

a e-mail: fanni.juranyi@psi.ch as fundamental sciences, it is very important to understand the Na-diffusion mechanism of the $\mathrm{Na}_{\mathrm{x}} \mathrm{CoO}_{2}$ compound.

Structurally, $\mathrm{Na}_{\mathrm{x}} \mathrm{CoO}_{2}$ consists of alternating $\mathrm{Na}$ and $\mathrm{CoO}_{2}$ layers. Within one unit cell $\mathrm{Na}$ can be located at one of the two crystallographic nonequivalent positions: either at $\mathrm{Na}_{1}$, located below and above the Co sites, or at $\mathrm{Na}_{2}$, located at the center of a Co trigonal prism [9]. For certain $x$ values (e.g. $x=1 / 3,1 / 2$ and 0.7$) \mathrm{Na}$ ions self-assemble into long-range ordered stable patterns [10], which is recognizable by weak superstructure peaks in the diffraction pattern. In addition, $\mathrm{Na}$ ions can rearrange at different temperatures, or undergo an order/disorder transition $[11,12]$.

\section{Experimental}

Polycrystalline samples of $\mathrm{Na}_{0.7} \mathrm{CoO}_{2}$ were prepared at the Paul Scherrer Institute (PSI), Switzerland as described in the Supplementary Material of Medarde et al. [1] according to the "rapid heat-up" technique proposed by Motohashi and co-workers [13]. The appropriated amounts of the starting materials $\mathrm{Na}_{2} \mathrm{CO}_{3}$ and $\mathrm{Co}_{3} \mathrm{O}_{4}(99.99 \%$ purity) were put into a pre-heated furnace $\left(790^{\circ} \mathrm{C}\right)$, held $15 \mathrm{~h}$ and cooled down to room temperature at $300^{\circ} \mathrm{C} / \mathrm{h}$. This procedure was repeated twice. The resulting material was immediately removed from the furnace and stored in an He-filled glove box. Neutron diffraction revealed the 


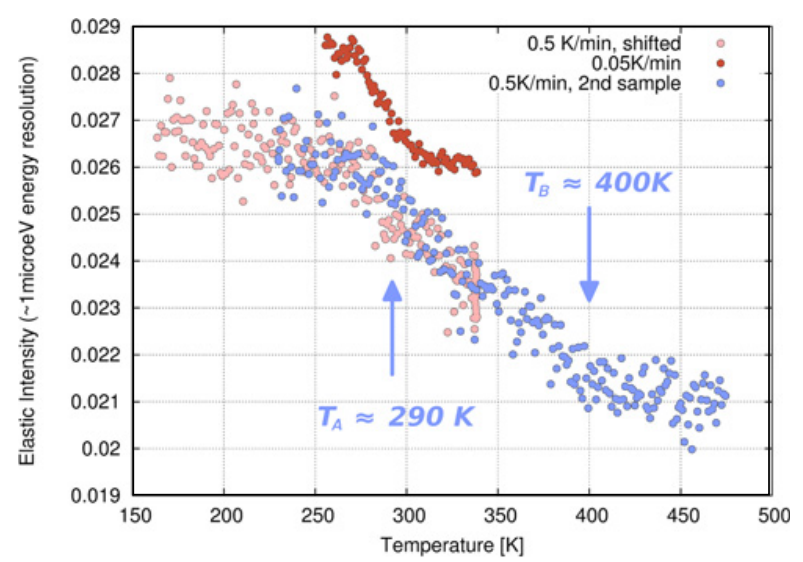

Figure 1. Elastic FWS of two different $\mathrm{Na}_{0.7} \mathrm{CoO}_{2}$ samples measured at IN16 (ILL). Counts from all detectors have been integrated (Q-range of 0.43 to $1.92 \AA^{-1}$ ). Arrows indicate the phase transition temperatures, which were found previously by means of neutron diffraction [1]. At about these temperatures changes in the spectra are observable. At $0.5 \mathrm{~K} / \mathrm{min}$ cooling rate the slope of the curve changes at both $T_{\mathrm{A}}$ and $T_{\mathrm{B}}$, while at $0.05 \mathrm{~K} / \mathrm{min}$ cooling rate two separate processes (two steps) are indicated by the plateau above $300 \mathrm{~K}$.

existence of a very small amount of $\mathrm{Na}_{2} \mathrm{CO}_{3}(<0.5 \%$ in weight). For the experiments the samples were enclosed in cylindrical, aluminum sample holders and sealed with lead.

Elastic fixed window scans (FWS) were performed to get an overview about the dynamics in the system as a function of temperature. In this type of measurement the intensity of the elastically scattered neutrons is measured. This signal is proportional to the portion of static scatterers in the material. Particles which are moving too slow in comparison with the observation time of the spectrometer contribute also to this signal. When the intensity of the elastically scattered neutrons decreases due to thermally activated motion, there is an increase of the neutron scattering intensity at non-zero energy transfer $E$. If the motion is diffusive, as expected for $\mathrm{Na}$ in $\mathrm{Na}_{\mathrm{x}} \mathrm{CoO}_{2}$ (from the diffraction experiment) [1], the quasielastic intensity increases. Therefore, also inelastic FWS were performed at low energy transfer.

The elastic FWS measurements (see Sect. 3.1) have been carried out at the IN16 backscattering spectrometer at the Institute Laue-Langevin (ILL), France with an energy resolution of $0.9 \mu \mathrm{eV}$. The incident and final neutron wavelength was $6.27 \AA$, corresponding to the backscattering position of the Si analyzer using the (111) reflection. Data has been taken during cooling using a constant temperature rate of 0.5 or $0.05 \mathrm{~K} / \mathrm{min}$. At some temperatures complete energy spectra $( \pm 15 \mu \mathrm{eV})$ were also recorded (see Sect. 3.2) using the Doppler drive for the monochromator.

For inelastic FWS (see Sect. 3.1) the high-resolution inverted time-of-flight (ToF) spectrometer MARS at PSI was used with an energy resolution of $\approx 13 \mu \mathrm{eV}$. At characteristic temperatures, i.e., below and above the phase transitions, significantly longer counting time (11.5 day per temperature) allowed for spectral analysis (see Sect. 3.2). Spectra were measured during heating.

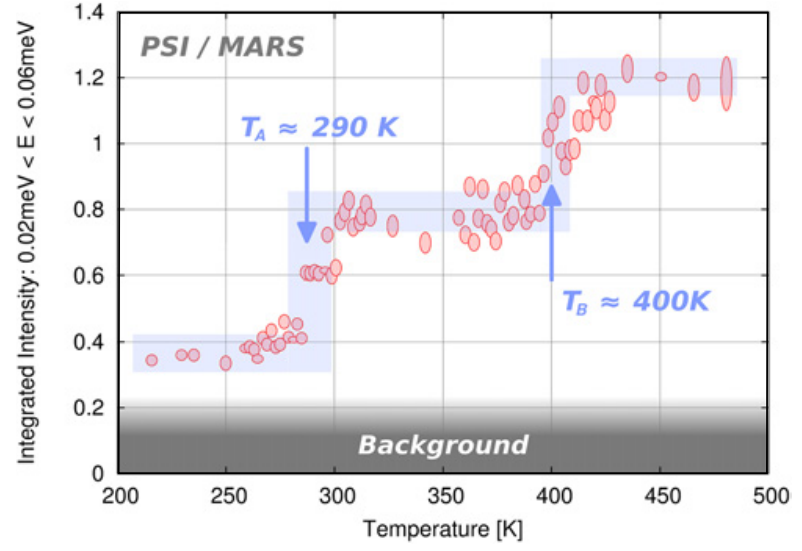

Figure 2. Inelastic FWS (merging several data sets) of $\mathrm{Na}_{0.7} \mathrm{CoO}_{2}$ measured at MARS at PSI. Data are integrated over an energy range of 20 to $60 \mu \mathrm{eV}$. Steps in the FWS at $\approx T_{\mathrm{A}}$ and $T_{\mathrm{B}}$ are clearly observable. The blue area is a guide to the eye. Background level is marked by the gray area. The vertical extension of the points corresponds to the statistical error (the different accuracy is caused by different counting times).

At MARS the scattered neutrons having a wavelength of $6.61 \AA$ were filtered by a mica analyzer using the (006) reflection. For the inelastic FWS, part of the spectra was integrated (between 20 and $60 \mu \mathrm{eV}$ ).

The phonon density of states (PDOS, see Sect. 3.3) was measured at the ToF spectrometer FOCUS at PSI using an incident wavelength of $\lambda_{i}=4.5 \AA$ and energy resolution of $\Delta E=140 \mu \mathrm{eV}$ (at the elastic line). Measurements were performed below and above the phase transitions temperatures $T_{\mathrm{A}}$ and $T_{\mathrm{B}}$. Counting times were about 12 hours for each spectrum. The aluminum sample container contributed significantly to the measured signal between 15 and $\approx 50 \mathrm{meV}$. Consequently, the empty container has been measured at the same temperatures with similar statistical accuracy as the sample itself to minimize the signal-to-noise ratio after background subtraction. In all experiments vanadium has been used to determine the energy resolution and to correct for the detector efficiency.

\section{Results}

\subsection{Overview of the dynamics: Elastic and inelastic fixed window scans}

At the previously observed structural phase transition temperatures (at $T_{\mathrm{A}}$ and $T_{\mathrm{B}}$ ) [1] $\mathrm{Na}^{+}$diffusion is expected to change markedly. Indeed, using a temperature ramp of $0.5 \mathrm{~K} / \mathrm{min}$ there is a change of slope in the FWS spectra measured at IN16 at both temperatures (see Fig. 1), indicating a change in the dynamics at $T_{\mathrm{A}}$ and $T_{\mathrm{B}}$. It looks like there would be one extended step (between $\approx T_{\mathrm{A}}$ and $\left.T_{\mathrm{B}}\right)$. However, at slower temperature rate and with corresponding better statistics (see red points in Fig. 1) a plateau is observable between $T_{\mathrm{A}}$ and $T_{\mathrm{B}}$. This means that two separate steps exist around the two transition temperatures corresponding to different processes. Steps in the elastic FWS should have their counterparts in the inelastic spectrum close to the elastic line if they are caused by diffusion. Therefore, energy spectra have been recorded 


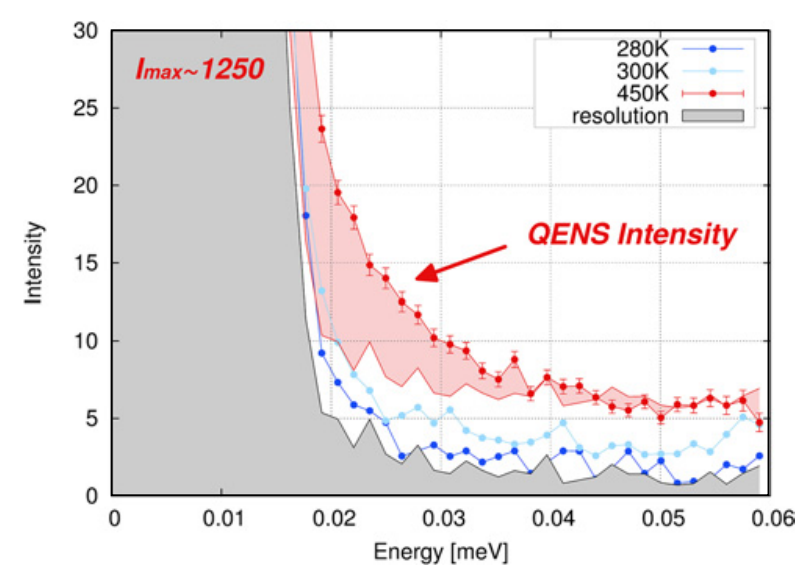

Figure 3. Quasielastic neutron scattering observed above $T_{\mathrm{B}}$ at the spectrometer MARS at PSI. Intensity of all detectors are integrated. The gray and red areas show the instrument resolution and the QENS signal, respectively.

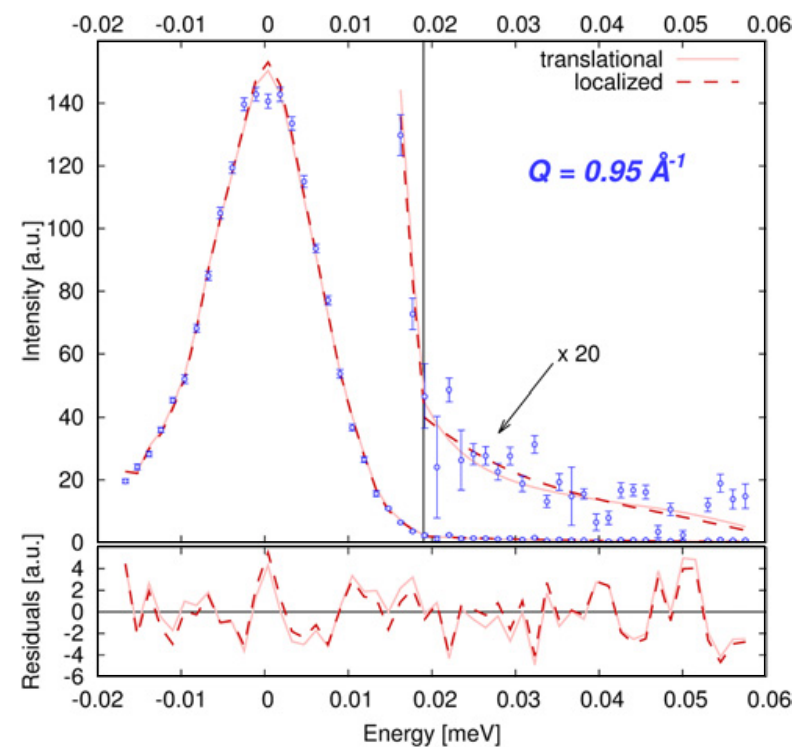

Figure 4. Energy spectrum of $\mathrm{Na}_{0.7} \mathrm{CoO}_{2}$ measured at $450 \mathrm{~K}$ at MARS at PSI. Vertical line shows the cut-off of the resolution function used in the convolution. Data at $Q=0.95 \AA^{-1}$ is shown, which is the most relevant regarding the type of the motion and the value of the diffusion coefficient. Solid and dotted lines represent two acceptable, model free fit of one Lorentzian. The parameters suggest long range translational or localized motion, respectively (see Fig. 5). Although the overall $\chi^{2}$ is slightly (7\%) higher for the long range translational diffusion, the data quality allows both possibilities.

on the same instrument at several temperatures between 255 and $340 \mathrm{~K}$. Unfortunately the total inelastic signal was only $10 \%$ above the background level, so a step in the integrated inelastic scattering is hardly visible. Contrary, at MARS the inelastic FWS showed clear steps around $T_{\mathrm{A}}$ and $T_{\mathrm{B}}$ (see Fig. 2).

\section{2. $\mathrm{Na}^{+}$diffusion: Quasielastic neutron Scattering}

The energy spectra collected at MARS show clear Quasielastic neutron Scattering (QENS) signal above $T_{\mathrm{B}}$

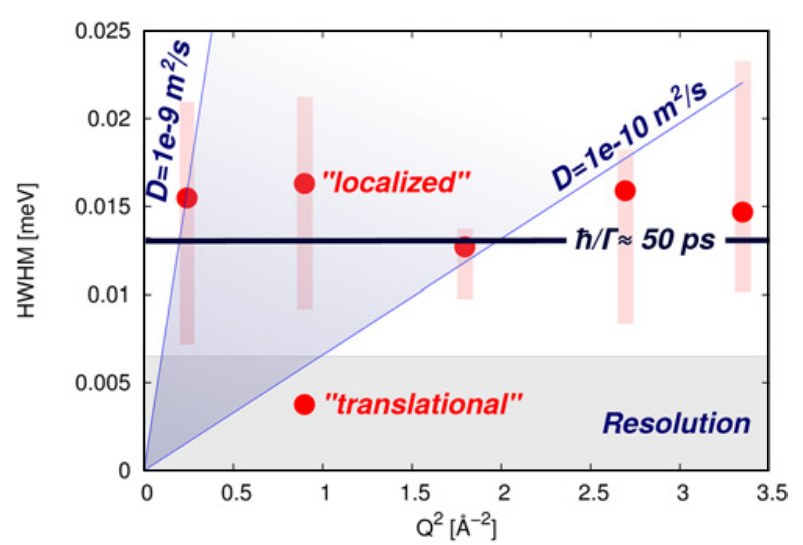

Figure 5. Width of the fitted Lorentzian function describing diffusion of $\mathrm{Na}$ in $\mathrm{Na}_{0.7} \mathrm{CoO}_{2}$ at $450 \mathrm{~K}$. Local fit minima with similar $\chi^{2}$ over a large range of HWHM values are indicated by the red bars. The best fits (smallest $\chi^{2}$ ) are shown by the red points. At $Q=0.95 \AA^{-1}$ two acceptable fit results are plotted, which corresponds to two possible diffusion models: long range translational and a localized diffusion. Fickian diffusion with extreme values of $D_{\mathrm{Na}}=1 e-9$ and $1 e-10 \mathrm{~m}^{2} / \mathrm{s}$ are shown. Line broadening corresponds to $50 \mathrm{ps}$ relaxation time is also shown.

(see Fig. 3). The spectra at 280 and at $300 \mathrm{~K}$ can be well fitted by a delta function at $E=0$ and a constant background, convoluted by the instrument resolution.

The QENS signal at 420 and $450 \mathrm{~K}$ can be fitted by an elastic line, one Lorentzian and constant background (see Fig. 4):

$$
\begin{aligned}
S(Q, E)^{\text {model }}= & A_{0}(Q) \times \delta(E)+A_{1}(Q) \\
& \times \frac{\hbar}{\pi} \frac{\Gamma(Q)}{\Gamma(Q)^{2}+E^{2}}+b g
\end{aligned}
$$

where $\Gamma(Q)$ is the $\mathrm{Q}$ dependent line width of the Lorentzian (HWHM). $S(Q, E)^{\text {model }}$ was numerically convoluted with the measured instrument resolution. Above $T_{\mathrm{B}}$ a $2 \mathrm{D}$ isotropic diffusion on a hexagonal lattice is expected [1]. The two nonequivalent $\mathrm{Na}$ sites $\left(\mathrm{Na}_{1}\right.$ and $\mathrm{Na}_{2}$ ), with different occupations, should be taken into account together with site blocking effects. Furthermore, since powder sample is used, the model function has to be averaged over all possible particle orientations. A similar model was developed for the diffusion of hydrogen in potassium-intercalated graphite [14].

In our case the fitted line width of a single Lorentzian is only roughly twice as broad as the instrument resolution, therefore the $2 \mathrm{D}$ behavior (which is manifested in a singularity at zero energy transfer) can not be resolved [15]. The model function under this circumstances can be approximated by a delta function (i.e. apparent increase of the height of the elastic line), and one Lorentzian (like if it would be a 3D motion, but the obtained 3D diffusion coefficient is $2 / 3$ of the real $2 \mathrm{D}$ one). Fitting two components for the two different Na sites would lead to an increased number of free parameters and would overdescribe the data.

Figure 5 shows the obtained line broadening (HWHM) of the Lorentzian at $450 \mathrm{~K}$. The fit parameters, especially 

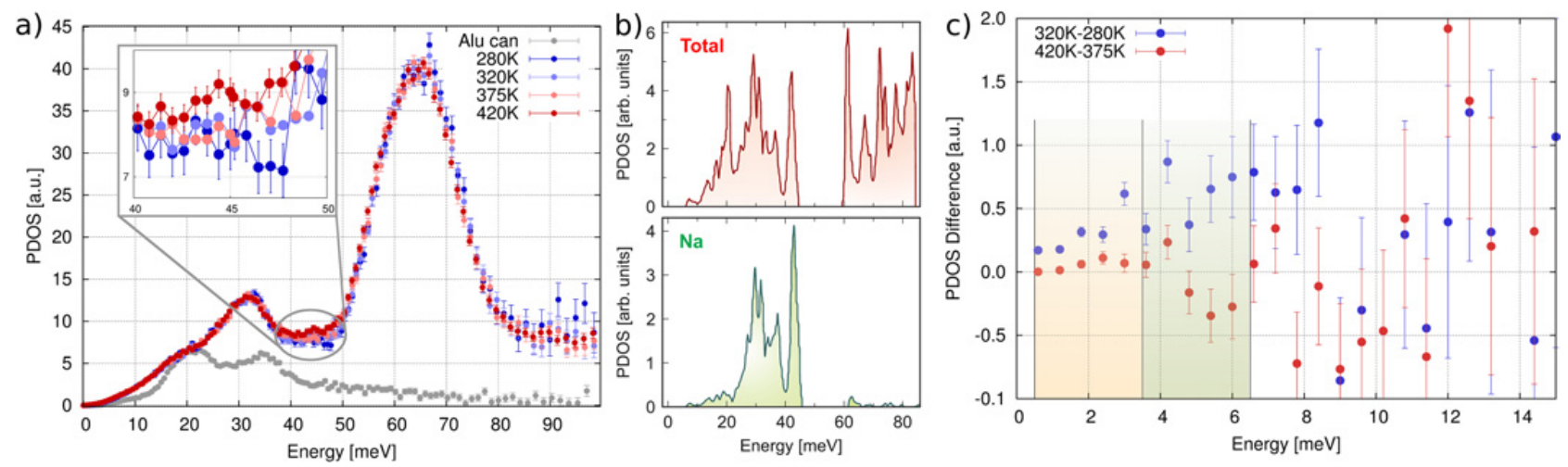

Figure 6. a) PDOS of $\mathrm{Na}_{0.7} \mathrm{CoO}_{2}$ around the transition temperatures, measured at the spectrometer FOCUS at PSI. Small changes in the Na PDOS are observed at $T_{\mathrm{A}}$ and $T_{\mathrm{B}}$. b) Calculated PDOS (redrawn after [16]). c) Differences in PDOS across the transitions. PDOS at low energies increases at $T_{\mathrm{A}}$, while remains unchanged by crossing $T_{\mathrm{B}}$.

the line width, depend strongly on the starting parameters. Therefore many starting configurations have been probed manually to obtain a best $\chi^{2}$ vs. HWHM curve for all Q values. Points at Fig. 5 are indicating the broadening for which the lowest $\chi^{2}$ was found, whereas the red bars the range, where the best found $\chi^{2}$ was not more than $2 \%$ higher than the minimum. The line broadening seems to be more or less independent of Q, which is a sign for localized motion. The corresponding area of the Lorentzian is smaller for the first two $Q$ values, which further support this interpretation. On the other hand, if the fit is started with an area similar to the ones at high Q, the obtained line broadening (marked with "translational" at Fig. 5) is significantly reduced. In doing so the corresponding $\chi^{2}$ is only $7 \%$ higher than the minimum. Also the residuals are similar for both fits (see Fig. 4), and the fitted curves are very close to each other compared to the scattering of the experimental data points. The data quality for the lowest $Q$ is even worse due to either smaller area or smaller broadening, therefore we have to conclude that the observed QENS signal can be caused either by a long range translational diffusion or by a localized motion.

In the first case we can estimate the magnitude of the diffusion coefficient $D_{\mathrm{Na}}$ as $5 \times 10^{-10} \mathrm{~m}^{2} \mathrm{~s}^{-1}$, which is valid for both 420 and $450 \mathrm{~K}$. The limiting slopes of $1 \times$ $10^{-10}$ and $1 \times 10^{-9} \mathrm{~m}^{2} \mathrm{~s}^{-1}$ in the Fickian approximation are plotted on Fig. 5. This estimated $D_{\mathrm{Na}}$ differs four orders of magnitude from the results of our muon-spin relaxation $\left(\mu^{+} \mathrm{SR}\right)$ spectroscopy experiments, where $D_{\mathrm{Na}}$ was found to be $3.80 \times 10^{-10} \mathrm{~cm}^{2} \mathrm{~s}^{-1}$ [2] at a similar temperature. If some kind of localized motion is assumed, the characteristic relaxation time $\tau=\hbar / E$ is in the order of $50 \mathrm{ps}$.

\subsection{Phonons: Inelastic neutron scattering}

PDOS was measured at various temperatures to complete the picture of $\mathrm{Na}^{+}$dynamics across the phase transitions: at $280 \mathrm{~K}$ (below $T_{\mathrm{A}}$ ), at 320 and $375 \mathrm{~K}$ (between $T_{\mathrm{A}}$ and $T_{\mathrm{B}}$ ) and at $420 \mathrm{~K}$ (above $T_{\mathrm{B}}$ ). The spectra show a small temperature dependency (see Fig. 6). The two spectra at 320 and $375 \mathrm{~K}$ are statistically identical. In the 3 different phases differences are visible between 30 and $50 \mathrm{meV}$.
These changes are clearly associated with $\mathrm{Na}$ vibrations based on the calculated (and neutron weighted) phonon spectra published by Jha et al. [16] (see Fig. 6). They also calculated the partial PDOS for both $\mathrm{Na}_{1}$ and $\mathrm{Na}_{2}$ sites. The small changes in the shape are not resolvable with the energy resolution of the instrument. But a small shift of the PDOS is expected for higher $\mathrm{Na}_{2}$ occupation with increasing temperature [1]. We observe higher intensity at higher energies in agreement with the expectation. Na-site occupancy is, however, only one aspect beside the changes in atomic distances and bond angles at $T_{\mathrm{A}}$ and $T_{\mathrm{B}}$. We speculate that the observed changes are rather connected to structural changes than to site occupation.

We can additionally see a tiny increase at energies below $\approx 5 \mathrm{meV}$ at crossing $T_{\mathrm{A}}$ (see Fig. 6) (at higher energies the statistical accuracy is not sufficient to detect such small changes). Probably this enhancement is observed as a step both in the elastic and inelastic FWS spectra (see Sect. 3.1).

Voneshen et al. [17] calculated significantly higher PDOS between $\approx 5$ and $20 \mathrm{meV}$ for $\mathrm{Na}_{0.8} \mathrm{CoO}_{2}$ if $\mathrm{Na}$ forms superstructure in comparison to the parent compound $\mathrm{NaCoO}_{2}$. They attributed this excess intensity to a low energy rattling mode, and its coupling to other low energy modes. In this energy range we could not detect any changes, and hence, no sign of different $\mathrm{Na}$ ordering in the different structural phases was found.

\section{Discussion and conclusions}

The $\mathrm{Na}^{+}$ion dynamics in $\mathrm{Na}_{0.7} \mathrm{CoO}_{2}$ has been investigated by neutron spectroscopy across the recently reported structural phase transition temperatures [1], between $\approx 160$ and $475 \mathrm{~K}$. At $T_{\mathrm{A}} \approx 290 \mathrm{~K}$ a stepwise decrease of the temperature dependent elastic intensity and a simultaneous increase of the low energy phonons could be observed. The coincidence of the onset of the diffusion $[1,2]$ and the appearance of low energy phonons suggest coupling between lattice vibrations and $\mathrm{Na}$ diffusion. It should be pointed out here that all the oxygen anisotropic Debye-Waller factors display an anomaly at $T_{\mathrm{A}}$ whereas their temperature dependence is smooth across $T_{\mathrm{B}}$ [1]. This 
suggest a possible role of the $\mathrm{O}$ phonon modes in the $\mathrm{Na}$ diffusion and calls for further investigations.

The small changes in the PDOS at both $T_{\mathrm{A}}$ and $T_{\mathrm{B}}$ could be assigned to $\mathrm{Na}^{+}$vibrations, which are rather caused by structural changes than by changes in the $\mathrm{Na}^{+}$ occupation.

Above $T_{\mathrm{B}}$ quasielastic scattering is detected with an energy resolution of $13 \mu \mathrm{eV}$. A simplified model has been used to describe the $2 \mathrm{D}$ diffusion on the hexagonal lattice. The estimated diffusion coefficient differs about four orders of magnitude from the results of $\mu^{+} \mathrm{SR}$ spectroscopy. For both techniques (QENS and $\mu^{+} \mathrm{SR}$ ) the observed motion above $T_{\mathrm{B}}$ is at the detection limit. Furthermore, $\mu^{+} \mathrm{SR}$ is model dependent. Although the low Q part of QENS data is model independent (Fickian limit), the data quality is bad. This gives some uncertainties in both cases, but the values definitely differ by magnitudes. In case of $\mathrm{Li}_{\mathrm{x}} \mathrm{CoO}_{2}$, the diffusion coefficients measured by means of nuclear magnetic resonance (NMR) spectroscopy at $400 \mathrm{~K}$ are about 4 magnitude less than calculated values [18], while the values obtained from $\mu^{+} \mathrm{SR}$ at $300 \mathrm{~K}$ agree well with the corresponding calculated values [18]. These discrepancies in the diffusion coefficients indicate that the $\mathrm{Na}^{+}$motion is more complex than thought. One realistic possibility is the presence of a second, fast localized type of motion beside the long range translational diffusion. If the QENS data is described by such type of motion, a relaxation time in the order of 50 ps can be obtained.

This work is based on experiments performed at the MARS and FOCUS spectrometers at the Swiss spallation neutron source SINQ, Paul Scherrer Institute, Villigen, Switzerland and at the IN16 spectrometer at the Institute Laue Langevin, Grenoble, France. JS was supported by MEXT KAKENHI Grant No. 23108003 and JSPS KAKENHI Grant No. 26286084.

\section{References}

[1] M. Medarde et al., Phys. Rev. Lett. 110, 266401 (2013)

[2] M. Månsson, J. Sugiyama, Phys. Scr. 88068509 (2013)

[3] V. Palomares et al., Energy Environ. Sci. 55884 (2012); S. Wenzel et al., Energy Environ. Sci. 4 3342 (2011); Y. Cao et al., Adv. Mater. 233155 (2011); S. Tepavcevic et al., ACS Nano 6530 (2012); M. D'Arienzo et al., Phys. Chem. Chem. Phys. 14 5945-5952 (2012)

[4] M. Roger et al.: Nature 445 (2007) 631

[5] D. J. P. Morris et al.: Phys. Rev. B 79 (2009) 100103.

[6] T. F. Schulze et al., Phys. Rev. Lett. 100026407 (2008)

[7] M. Weller et al., Phys. Rev. Lett. 102056401 (2009)

[8] M.-H. Julien et al., Phys. Rev. Lett. 100096405 (2008)

[9] Y. Wang, Y. Ding and J. Ni, J. Phys.: Cond. Mat. 21 035401 (2009)

[10] H. W. Zandbergen et al., Phys. Rev. B. 70024101 (2004)

[11] Y. Hinuma, Y.S. Meng, G. Ceder, Phys. Rev. B 77 224111 (2008)

[12] D. Igarashi,Y. Miyazaki, T. Kajitani, K. Yubuta, Phys. Rev. B 78184112 (2008)

[13] T. Motohashi et al., Appl. Phys. Lett. 79, 1480 (2001)

[14] J. Purewal et al., J. Chem. Phys. 137224704 (2012); http://thesis.library.caltech.edu/5574/ 7/Purewal_Thesis_Ch5.pdf

[15] R. E. Lechner, Solid State Ionics 77 280-286 (1995)

[16] P.K. Jha et al., Physica B 366, 153 (2005)

[17] D. J. Voneshen et al., Nature Materials 12, 1028-1032 (2013)

[18] J. Sugiyama et al. Phys. Rev. Lett. 103, 147601 (2009) 